\title{
Widersprüchliche Verkehrsbezeichnungen auf Fertigpackungen - Systematischer Versuch der Verbrauchertäuschung oder korrekte Kennzeichnung?
}

\author{
Dr. H. Kirchhoff, A. Orellana und M. Leidreiter \\ Niedersächsischen Landesamtes für Verbraucherschutz und Lebensmittelsicherheit (LAVES), Lebensmittelinstitut Oldenburg, \\ Martin-Niemöller-Str. 2, 26133 Oldenburg
}

\section{Zusammenfassung/Erläuterung}

Im Handel werden mit zunehmender Häufigkeit Fertigpackungen angeboten, die lediglich auf einer Packungsseite die vollständige und zutreffende Verkehrsbezeichnung des Packungsinhaltes tragen, während auf allen anderen Seiten eine unvollständige, meist ein hochwertigeres Produkt suggerierende Bezeichnung angebracht ist.

Ein Beispiel ist eine Kartonverpackung mit Hähnchenbrustfilets mit 8\% Flüssigwürzung. Auf 4 Seiten des Kartons ist die Bezeichnung „Hähnchenbrust-Filets“ aufgedruckt. Lediglich auf einer Seite des Kartons findet sich die korrekte Verkehrsbezeichnung „Hähnchenbrust-Filets Mit 8\% Flüssigwürzung, aus Hähnchenbrustfilet, küchenfertig, tiefgefroren, einzeln entnehmbar“. Da es sich hier um die Seite der Verpackung handelt, auf der sich auch die anderen im gleichen Sichtfeld mit der Verkehrsbezeichnung anzugebenden Kennzeichnungselemente befinden, ist die Fertigpackung nach Meinung der Hersteller damit korrekt gekennzeichnet.

Faktisch befindet sich jedoch auf vier Verpackungsseiten eine nicht zutreffende Bezeichnung, denn die Angabe „mit 8\% Flüssigwürzung“ ist als Teil der Verkehrsbezeichnung anzusehen und nicht als Zusatz zu dieser. Somit trägt die Fertigpackung zwei unterschiedliche Verkehrsbezeichnungen, die für verschiedene Produkte stehen.

Weitere Beispiele sind Fertigpackungen mit dem Produkt „Hähnchenschenkel mit Rückenstück“, bei denen die Worte „mit Rückenstück“ nur auf der einen Seite angegeben sind, oder deutlich räumlich getrennt von dem Wort „Hähnchenschenkel“. Dabei ist die Bezeichnung „Hähnchenschenkel mit Rückenstück“ sogar eine in Rechtsvorschriften festgelegte Verkehrsbezeichnung (vgl. Art. 1 Nr. 2 f VO (EWG)1538/91).
Obwohl die Rechtsprechung des EuGH verbreitet derart ausgelegt wird, dass für den interessierten und verständigen Verbraucher die Angabe der Verkehrsbezeichnung auf nur einer Packungsseite ausreichend ist, kann eine solche Praxis aus hiesiger Sicht durchaus als irreführend beurteilt werden. Durch weitere Beispiele soll gezeigt werden, wie von Herstellerseite offensichtlich versucht wird, die diesbezügliche Rechtsprechung des EuGH und das ihr zu Grunde liegende Verbraucherleitbild zum eigenen Vorteil und zum (Informations-)Nachteil großer Teile der Verbraucherschaft auszunutzen.

Entsprechend den Erwägungsgründen der Lebensmitteletikettierungsrichtlinie soll u.a. ,jede Regelung der Etikettierung von Lebensmitteln vor allem der Unterrichtung und dem Schutz der Verbraucher dienen“. Außerdem „, müssen die Regeln für die Etikettierung das Verbot enthalten den Käufer zu täuschen“ und „um wirksam zu sein, muss dieses Verbot auf die Aufmachung der Lebensmittel ausgedehnt werden“.

Unter diesen Aspekten soll an Hand der gezeigten Beispiele die Frage diskutiert werden, nach welchen Kriterien eine Beanstandung der Gesamtaufmachung als irreführend i.S. des §11 Abs. 1 Nr. 1 LFGB gerechtfertigt ist, und ob solche Beanstandungen zurzeit rechtlich durchsetzbar sind?

\section{Beschluss (einstimmig)}

DiebestehendeProblematikbei der kennzeichnungsrechtlichen Beurteilung der Aufmachung von Fertigpackungen mit Lebensmitteln wird zur Kenntnis genommen. Die Gesamtaufmachung ist jeweils im Einzelfall hinsichtlich einer Täuschungsmöglichkeit zu beurteilen und ggf. zu beanstanden! Ergänzend wird auf den Beschluss zum Beitrag von Baumeister et al. hingewiesen. 